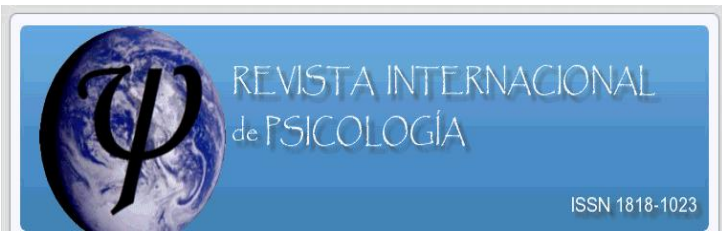

\title{
LA RELACIÓN PERSONA-ENTORNO COMO FUENTE GENERADORA DE ESTRÉS ACADÉMICO
}

\author{
Dr. Arturo Barraza Macías ${ }^{1}$
}

Como hipótesis central del modelo sistémico cognoscitivista, elaborado para el estudio del estrés académico (Barraza, 2006), afirmé que una demanda del entorno sólo se convierte en estresor tras la valoración cognitiva que hace el sujeto de ella, sin embargo, creo necesario realizar algunas precisiones al respecto para no dejar una impresión errónea, ya que es posible que alguno de los lectores se haya preguntado ¿y dónde queda el papel del entorno? Esta pregunta, nada ociosa y si muy pertinente, adquiere relevancia si se toma en cuenta que el modelo elaborado se inscribe en el Programa de Investigación Persona-Entorno (Barraza, 2007a) y tal pareciera que el entorno ha desaparecido del escenario. Nada más lejos de la verdad y para corregir esa impresión es que se realiza el presente trabajo.

\section{El papel del entorno en el estrés académico}

El proceso a través del cual una demanda del entorno se constituye en estresor se explica por la interacción entre la persona y el entorno, pero una interacción donde el entorno presente ciertas características que lo hacen potencialmente generador de estrés y que la persona presente ciertas características que lo conduzcan a una valoración cognitiva, potencialmente negativa, de las demandas que le presenta el entorno.

Un entorno potencialmente generador de estrés es un entorno novedoso en sus demandas, exageradamente demandante, altamente impredecible, escasamente controlable, con demandas de alta duración y elevada intensidad. Ante estas características del entorno una persona puede realizar una valoración cognitiva potencialmente negativa cuando perciba en ella un bajo nivel de autoeficacia (Bandura, 1997 y 1999) y manifieste una centración en las atribuciones causales externas (Weiner, 1974 y 1986).

Esta relación entre las características del entorno y las características de las personas constituye un proceso adaptativo, donde la persona se adapta al entorno. Esta relación, de carácter adaptativa, es lo que realmente define el origen de un estresor, como se puede observar en el siguiente ejemplo:

La Escuela Preparatoria Izcalli es una institución altamente demandante, les exige a sus alumnos múltiples tareas y actividades escolares y realiza cada semana evaluaciones de control. En esta institución, alumnos como Juan manifiestan estrés académico ya que Juan se considera ineficaz o poco capaz para estudiar cada semana para ocho exámenes, por lo que la valoración que realiza de los exámenes lo conduce a considerarlos como una amenaza que le genera estrés.

Con este ejemplo basta para ilustrar, por el momento, como las características del entorno interactúan con las características de la persona para originar un estresor. En ese sentido, la generación de un estresor se vuelve un proceso relativo e interactivo,

\footnotetext{
${ }^{1}$ Universidad Pedagógica de Durango. Correos electrónicos: tbarraza@terra.com.mx barraza_upd@terra.com.mx
} 
Relativo por que cierta característica específica del entorno, como es el caso de un maestro altamente demandante, no necesariamente producirá estrés en sus alumnos, e interactivo por que dependerá de las características de cada alumno al relacionarse con esa característica del entorno.

Relativo por que cierta característica de la persona, como sería el caso de la percepción de un bajo nivel de autoeficacia, no necesariamente producirá estrés en el alumno, e interactivo por que dependerá de las características de la institución donde esté inscrito, del grupo con el que comparta la clase o de los maestros que le impartan clases.

\section{Las características del entorno potencialmente generadoras de estrés}

En la línea discursiva que se ha venido desarrollando hasta este momento es necesario entender al entorno como el conjunto de situaciones, que de carácter normativo o contingente, definen y estructuran las relaciones de los diferentes agentes involucrados en un espacio organizacional delimitado. Con esta definición, de carácter psicosocial, se establece una distancia con ciertos enfoques de la perspectiva organizacional que centran su atención en aspectos físicos del entorno (v. gr. iluminación) o con posturas como la de Trianes (2002) quien al abordar el papel de la familia en el estrés infantil hace referencia a un entorno empobrecido, entendiéndolo como las condiciones objetivas y materiales que definen un entorno físico.

Desde esta perspectiva psicosocial se puede afirmar que las características del entorno, potencialmente generadoras de estrés, no han sido lo suficientemente estudiadas, sin embargo algunos autores como Amigo (2000) y Cruz y Vargas (2001) refieren ciertas características que deben tener las situaciones para ser generadoras de estrés. Por otra parte, en los trabajos realizados con alumnos de maestría (Barraza 2007b y 2007c) he hipotetizado sobre las características del entorno que explican potencialmente las diferencias en el estrés académico de diferentes entornos institucionales.

Con base en estos antecedentes me permito construir una tipología de características de los entornos que son potencialmente generadoras de estrés, y en este caso, de estrés académico. Esta tipología permite responder a la siguiente pregunta ¿Qué es lo que hace a una institución, a un grupo, a un maestro o a una situación de aprendizaje potencialmente generadora de estrés?

La respuesta a esta pregunta gira alrededor de las seis características que definen los tipos de entornos por el tipo y/o la cantidad de las demandas que presentan:

Entornos exageradamente demandantes,

Entornos con demandas novedosas.

Entornos altamente impredecibles en las demandas,

Entornos escasamente controlables en sus demandas, 
Entornos con demandas de alta duración y

Entornos con demandas de elevada intensidad.

A continuación se describirán cada uno de ellos y sus características que los hacen potencialmente generadores de estrés.

a) Entornos exageradamente demandantes,

En la Universidad Massada el trabajo que realiza normalmente un alumno de postgrado es el siguiente: lectura de dos o tres textos diarios (aproximadamente 45 páginas), realiza fichas resumen por cada uno de los textos leídos, prepara una exposición por lo menos una vez al mes, elabora un ensayo por cada unidad temática abordada, lee durante el semestre por lo menos dos libros como complemento a la antología de lecturas y elabora un trabajo final que puede ser un ensayo, un diseño curricular, una práctica investigativa o el diseño de una revista.

En la Universidad Sahuatoba el trabajo que realiza normalmente un alumno de postgrado es el siguiente: la lectura de un texto de aproximadamente 15 páginas a la semana, prepara una exposición al semestre y al final presenta un trabajo por equipo.

En estos dos casos se puede observar como las demandas que el entorno institucional les presenta a sus alumnos son cuantitativamente diferentes. En ese sentido, un entorno altamente demandante se vuelve un entorno potencialmente generador de estrés, mientras que el entorno que es pobremente demandante es menos probable que genere estrés en sus alumnos.

Un entorno altamente demandante significa mayores posibilidades de estrés, pero a la vez, mayores posibilidades de aprendizaje para sus alumnos, sin embargo es necesario tener cuidado que la multiplicidad y variedad de demandas no provoquen un alto nivel de estrés que en lugar de permitir al alumno activarse para lograr el aprendizaje lo conduzca al bloqueo y a la inhibición de la acción.

b) Entornos con demandas novedosas

Elena acaba de salir de una institución altamente conservadora y tradicional. En ella los maestros todavía dictan la cátedra y se valora el aprendizaje memorístico. Ahora, acaba de entrar a otra institución donde los maestros le exigen que lea ella directamente los textos, que analice ei contenido, que reflexione sobre las implicaciones del tema, que valore su pertinencia para su contexto y que al final realice una crítica del contenido.

¿Cómo son valoradas estas demandas por Elena? Tal vez en el caso de otros alumnos éstas sean solamente unas actividades escolares más, pero en el caso de Elena esta forma de trajo se vuelve un estresor. El hecho de que todas estas demandas sean novedosas para Elena, que no está acostumbrada a esta forma de trabajo, hace que estas demandas sean valoradas como estresores y provoquen en ella el estrés académico. 
c) Entornos altamente impredecibles en las demandas.

El maestro de la asignatura de Química en la Escuela Preparatoria Izcalli inicia el semestre presentándoles a los alumnos el contenido del curso, planea en conjunto con ellos las actividades a realizar, el tipo de trabajo que se desarrollará y la forma y los momentos de evaluación. Esta programación es respetada por el maestro y cualquier cambio es discutido primero con el grupo.

El maestro de la asignatura de Cálculo, en la Escuela Preparatoria Izcalli, no presenta a los alumnos el contenido de la materia ni les informa las actividades a realiza, ni el tipo de trabajo a desarrollar ni la forma y los momentos de evaluación. En cada clase improvisa lo que se trabaja, las actividades las genera en ese momento según su estado de ánimo, el tipo de trabajo a realizar lo cambia de una sesión a otra y cuando se le ocurre llega y aplica algún examen.

Como se puede observar, los dos casos arriba mencionados son diametralmente opuestos y sin lugar a dudas las consecuencias también. Un entorno normado donde el alumno sabe que sigue, que tipo de trabajo necesita hacer y cuando va a presentar un examen es un entorno que difícilmente provocará estrés, sin embargo un entorno donde priva la improvisación, el estado de ánimo o la ocurrencia hace que cada demanda se constituya potencialmente en un estresor.

d) Entornos escasamente controlables en sus demandas

El maestro de la asignatura de Psicología Evolutiva II de la Licenciatura en Psicología de la Universidad Massada le presenta a los alumnos las partes y porcentajes que comprende la evaluación del semestre: $10 \%$ de la calificación se adquieren teniendo como mínimo el 90\% de asistencia, el 30\% corresponde a las fichas resumen que entregaran cada semana, el 20\% corresponde al trabajo por equipo que realizarán al final y el $40 \%$ a la participación en clase. El alumno conociendo estos elementos y sus respectivos porcentajes puede ir controlando durante el semestre el trabajo que realiza para alcanzar a promover la asignatura, $v$. gr. Juan trata de no faltar por lo que sabe que tiene un $10 \%$ asegurado, entrega las fichas resumen lo que le agrega un $20 \%$ a su evaluación, trata de asegurar la calificación mínima aprobatoria en las participaciones para obtener la mitad del porcentaje $20 \%$ y sabe que entonces solamente le falta un $10 \%$ para aprobar y este lo puede lograr con una calificación mínima aprobatoria en el trabajo final. Bajo estas condiciones Juan puede dosificar su esfuerzo y el trabajo que desarrolla.

El maestro de la asignatura de Dinámicas Grupales de la Licenciatura en Psicología de la Universidad Massada les informa a sus alumnos que la calificación que obtenga depende de la participación que tengan en clase (sin aclarar los criterios de evaluación de dicha participación) y que en su momento decidirá si aplica o no un examen. En estas condiciones los alumnos no saben como manejar sus tiempos y su 
esfuerzo para aprobar la asignatura, en otras palabras ellos no pueden controlar su proceso de acreditación.

En este segundo ejemplo se puede observar un claro, y recurrente, entorno donde el alumno no puede controlar su proceso de aprendizaje o evaluación y todo queda supeditado a la voluntad y criterio del profesor. En esta situación, las actividades o trabajos que solicita un profesor pueden sen valorados como altamente estresantes.

e) Entornos con demandas de alta duración.

La maestra de la asignatura de Política Educativa en el primer semestre de maestría de la Universidad Sahuatoba le pide a sus alumnos que lean para cada sesión cuatro lecturas de aproximadamente 15 páginas cada una y como control de lectura les solicita que le elaboren un mapa conceptual sobre el contenido de cada texto. Para un alumno no acostumbrado a leer esta tarea significa un esfuerzo extra y le dedica varias horas de su tiempo a la lectura y otro tanto de tiempo a la elaboración de los mapas conceptuales.

Esta demanda, más allá de la presión originada al inicio, suele ser valorada como estresor en el momento en que esta actividad se prolonga por todo el semestre y el cansancio va haciendo mella en los estudiantes.

f) Entornos con demandas de elevada intensidad

La maestra de la asignatura de Desarrollo Infantil III de la Licenciatura en Educación Preescolar de la Escuela Normal Michilia les pide a sus alumnos como evaluación final del semestre la elaboración de un video sobre el desarrollo moral del niño. Esta actividad implica que los alumnos deben: a) conseguir quien les tome el video y se los edite, b) aprender como se hace un guión para un video, c) conocer el contenido, en este caso el desarrollo moral del niño, d) elaborar el guión del video, e) seleccionar los escenarios pertinentes para la toma del video, f) guiar la toma de escenas, g) supervisar la edición del video y h) supervisar la versión final del video.

La maestra de la asignatura de Psicomotricidad de la Licenciatura en Educación Preescolar de la Escuela Normal Michilia les pide a sus alumnos como evaluación final del semestre la elaboración de un ensayo donde expliquen la importancia de la psicomotricidad para el aprendizaje del niño.

Como se puede observar las dos actividades de evaluación final tienen diferente grado de intensidad si se toma en cuenta que las actividades que deben realizar en cada caso difieren substancialmente, por lo que en el primer caso se observa una demanda de trabajo con mayor nivel de intensidad. 


\section{Las características de la persona potencialmente generadoras de estrés}

Como afirmé anteriormente el proceso cognoscitivo que hace posible que las demandas del entorno sean valoradas como estresores es la valoración cognitiva que las personas hacen de las mismas. En ese sentido cabe preguntarse ¿Por qué una persona puede valorar, más allá de las características del entorno, como una amenaza o un peligro ciertas demandas del entorno? La respuesta es simple: por que cree que estas demandas desbordan los recursos de que dispone.

Ante esa respuesta ahora la pregunta clave es ¿por qué cree esa persona que dichas demandas desbordan los recursos de que dispone? La respuesta a esta pregunta la he buscado en otros procesos cognoscitivos: la autoeficacia y a las atribuciones causales (Barraza, 2007d).

\section{a) Autoeficacia}

La consecución de determinadas metas constituye un ideal común a todas las personas, una motivación intrínseca que lleva al ser humano a emprender conductas específicas en función de los logros que éste pretende alcanzar. Mas no basta con conocer con claridad aquello que deseamos lograr, ni tampoco el mejor medio para poder conseguirlo. No basta con ser capaz de; es preciso juzgarse capaz de utilizar las capacidades y habilidades personales ante circunstancias muy diversas.

La percepción de las personas acerca de su propia eficacia se alza como un requisito fundamental para desarrollar con éxito las acciones conducentes al logro de los objetivos personales.

Dicha autopercepción, denominada autoeficacia, ejerce una profunda influencia en la elección de tareas y actividades, en el esfuerzo y perseverancia de las personas cuando se enfrentan a determinados retos e incluso en las reacciones emocionales que experimentan ante situaciones difíciles. En definitiva, las creencias de autoeficacia representan un mecanismo cognitivo que media entre el conocimiento y la acción y que determina, junto con otras variables, el éxito de las propias acciones.

Esta Teoría ha sido formulada por Bandura (1977, 1982, 1997 y 1999) y su núcleo teórico básico se constituye por los siguientes supuestos:

1) Las expectativas de eficacia personal determinan el esfuerzo que las personas emplean para realizar una tarea específica así como su grado de perseverancia cuando se enfrentan a situaciones difíciles (Bandura, 1977).

2) La autopercepción de la eficacia personal determina, en cierto modo, los patrones de pensamiento, las acciones y las reacciones emocionales del ser humano (Bandura, 1982).

3) Las personas que gozan de un alto nivel de autoeficacia son más eficaces y consiguen más éxitos que aquellas otras con expectativas bajas de eficacia personal. (Bandura, 1997). 
4) La percepción de la eficacia personal para afrontar las múltiples demandas de la vida cotidiana afecta al bienestar psicológico, al desempeño y al rumbo que toma la vida de las personas (Bandura, 1999).

El constructo de la autoeficacia, sus características, su medida y su repercusión en la conducta humana ha suscitado el interés de investigadores de todos los ámbitos, mayoritariamente en el campo de las ciencias de la salud y en la investigación educativa, con especial hincapié en el área de la motivación académica.

La investigación educativa ha realizado múltiples estudios sobre las creencias de autoeficacia, especialmente en el área de la autorregulación y la motivación académica. En este sentido, los investigadores han abordado tres aspectos relacionados con el constructo de la autoeficacia (Prieto, 2003):

- autoeficacia y elección de carrera

- autoeficacia del profesor y práctica docente

- autoeficacia de los alumnos, motivación y rendimiento académico

Personalmente creo que este constructo puede ayudar a explicar el por qué la persona realiza una valoración cognitiva negativa de las demandas del entorno. En ese sentido, la persona cree que no tiene los recursos para afrontar la demanda del entorno por que se juzga poco capaz o incapaz de actuar como lo solicita la demanda, ya sea por que considera que no tiene las habilidades o las destrezas necesarias o por que considera que no posee los conocimientos suficientes.

Juanita, alumna de la Escuela Preparatoria Izcalli, se entera por parte del maestro de la asignatura de Química que para poder acreditar el semestre necesita obtener un nueve en la última evaluación. A pesar de que faltan quince días para el último examen, tiempo suficiente para estudiar, Juanita no solamente no estudia sino que manifiesta síntomas inequívocos de estrés y cuando se le cuestiona el por qué de ese estrés manifiesta tajantemente "ya reprobé; soy muy bruta (sic) no voy a poder sacar nueve".

b) Atribuciones causales

"La atribución es el proceso por el que las personas interpretan su comportamiento y el de otros, y les asignan causas. El concepto se deriva del trabajo de Fritz Heider y lo han ampliado y mejorado Harold Kelley y otros". (Davis y Newstrom, 2003; pg. 170).

Los enfoques basados en la atribución causal (Heider, 1958; Kelley, 1972; Weiner, 1974 y 1986) se interesan por conocer la fuerza motivacional de la reflexión mental que sigue a un evento. Los humanos tendemos a buscar las causas explicativas de la conducta, de manera especial cuando los resultados obtenidos no coinciden con las expectativas previas. 
Los teóricos de la atribución coinciden en los aspectos cognitivos y racionales de la conducta voluntaria, pero el interés no se centra ni en la determinación de intenciones ni en su cumplimiento, sino que se localiza específicamente en los argumentos explicativos que dan las personas acerca del por qué de los resultados obtenidos, tanto si éstos son percibidos como éxitos o, por el contrario, como fracasos.

Se parte de dos supuestos básicos: 1) que cualquier atribución humana obedece a unas determinadas reglas y 2) que las atribuciones causales establecidas van a influir sobre el desarrollo de nuevos comportamientos y futuras expectativas, repercutiendo, en definitiva, en el establecimiento y selección de metas futuras (Weiner, 1986).

Por atribución causal se puede entender aquella interpretación que el individuo realiza respecto a los elementos que tienen un cierto grado de responsabilidad sobre el resultado de una acción cognitiva o conducta. La atribución sería aquella inferencia que el individuo realiza para determinar qué ocasiona la situación. Su importancia radica en que dependiendo de esta consideración, el sujeto va a actuar de una u otra manera, evitando o fomentado esta causa. atribución:

Echevarria (citado por Morgana, 2004) destacó cuatro principios de la

La atribución causal es una actividad cotidiana.

Las atribuciones no son exactas, ya que existen errores atribucionales.

La conducta depende de cómo las personas perciben e interpretan los hechos.

Los procesos atribucionales cumplen una función adaptativa.

Esta teoría ha servido de base a múltiples investigaciones, pudiéndose identificar tres líneas de investigación (Barraza y Ontiveros, 2006):

1. Una primera línea de investigación es sobre el éxito y el fracaso escolar, de hecho esta línea se constituye en su principal línea de investigación. Una variante de esta línea de investigación relaciona el rendimiento escolar con las atribuciones causales y otros factores motivaciones como sería el caso de las metas académicas,

2. En una segunda línea de investigación, bajo una perspectiva psicológica, se busca encontrar la relación de las atribuciones causales con otros factores motivacionales, cognitivos o metacognitivos.

3. Una tercera línea de investigación, de carácter emergente, es la elección de carrera profesional.

Creo que la atribución causal que un alumno le atribuye al logro o no logro de una demanda es lo que posibilita que la considere o no estresor. Cuando el logro, o la respuesta a una demanda del entorno, considera el alumno es atribuible a personas o situaciones ajenas a él (atribuciones externas) es más probable que el alumno crea que 
no puede afrontarla y en ese sentido realiza una valoración cognitiva de carácter negativo que lo lleva a considerar a esa demanda como un estresor.

Cuando el logro o la respuesta a una demanda del entorno, considera el alumno es atribuible a él mismo (atribuciones internas), entra el juego la percepción de su propia autoeficacia para enfrentar dicha demanda; si el alumno se considera poco capaz para enfrentar dicha demanda es más probable que el alumno realice una valoración cognitiva de carácter negativo que lo lleva a considerar a esa demanda como un estresor.

\section{Combinaciones probables entre las características del entorno y las características de las personas que generan estrés académico}

$\mathrm{Si}$ el origen del estrés académico se encuentra en la relación entre las características de las personas y las características del entorno, la tarea de identificar las fuentes del estrés puede aparentar ser una tarea inocua si se toma como base el carácter idiosincrático del proceso, sin embargo, creo se pueden establecer ciertas generalizaciones a partir de posibles combinaciones que potencialmente son generadoras de estrés.

Las combinaciones de ciertas características del entorno con ciertas características de las personas aumentan significativamente la probabilidad de manifestar estrés académico. A continuación presento varias combinaciones en donde la probabilidad de que se manifieste el estrés académico es muy alta:

\section{Combinación Uno:}

Entorno exageradamente demandante + Entorno con demandas altamente impredecibles + Persona generadora de atribuciones causales externas = Estrés Académico.

\section{Combinación Dos:}

Entorno con demandas novedosas + Entorno con demandas de alta duración + Entorno con elevada intensidad en sus demandas + Persona generadora de atribuciones causales internas + Persona con la percepción de un bajo nivel de autoeficacia = Estrés Académico.

\section{Combinación Tres:}

Entorno exageradamente demandante + Entorno con demandas novedosas + Persona generadora de atribuciones causales internas + Persona con la percepción de un bajo nivel de autoeficacia = Estrés Académico.

\section{Combinación Cuatro}

Entorno altamente impredecible en sus demandas + Entorno con demandas de alta duración + Entorno con demandas de elevada intensidad + Persona generadora de atribuciones causales externas $=$ Estrés Académico

\section{Combinación Cinco}

Entorno escasamente controlables + Entorno altamente impredecible + Persona generadora de atribuciones causales internas + Persona con una alta percepción de su nivel de autoeficacia $=$ Estrés Académico. 


\section{CONCLUSIONES}

Las combinaciones anteriormente descritas, por mencionar solo las más claras e identificables a primera vista, aumentan significativamente la probabilidad de que se presente estrés académico; en ese sentido se puede afirmar que, dichas combinaciones, aumentan la probabilidad de que las demandas del entorno sean valoradas por la persona como estresores. Con la presentación de estas combinaciones cierro, por el momento, la discusión de cómo la relación entre las características del entorno y las características de la persona son la fuente generadora del estrés académico.

\section{REFERENCIAS}

Amigo V. I. (2000), El precio biológico de la civilización, Madrid, España, Celeste Ediciones S.A.

Barraza M. A. (2006), Un modelo conceptual para el estudio del estrés académico, en la Revista Electrónica de Psicología Iztacala, Vol. 9. No. 3, pp. 110-129 (on line)

Barraza M. A. (2007a), El campo de estudio del estrés: del Programa de Investigación Estímulo-Respuesta al Programa de Investigación Persona-Entorno, Revista Internacional de Psicología, No. 2, Vol. 8 (on line)

Barraza M. A. (2007b), El estrés académico de los alumnos de los postgrados en educación, Tesis Doctoral, Durango, Instituto Universitario Anglo Español.

Barraza M. A. (2007c), El estrés académico de los alumnos de las maestrías en educación de la región laguna (Durango-Coahuila), disponible en la Biblioteca Virtual de Psicoactiva, en la sección artículos científicos y en Monografías.com. (recuperado el 27/08/2005)

Barraza M. A. (2007d), Estrés académico. Líneas de investigación para el desarrollo de un modelo explicativo, disponible en ILUSTRADOS.COM, (recuperado el 30/08/2005)

Barraza M. A. y Ontiveros H. V. C. (2006), Atribuciones causales a la elección de carrera. Caso: Licenciatura en Intervención Educativa, en la Revista Divulgación, Año 5, No. 7,pp. 19-23

Bandura A. (1977): Self-efficacy: Toward a unifying theory of behavioral change en Psychological Review, No. 84, vol.2,pp. 191-215.

Bandura A. (1982), Self-efficacy mechanism in human agency, en American Psychologist, No.37, Vol.2, pp. 122-147.

Bandura A. (1997), Self-efficacy: The exercise of control. New York, USA, N.H. Freeman.

Bandura A. (1999), Autoeficacia: Cómo afrontamos los cambios de la sociedad actual, Bilbao, España, Desclée Brouwer.

Cruz M. C. y Vargas F. L. (2001), Estrés; entenderlo es manejarlo, México, Alfa Omega.

Davis K. y Newstrom J. W. (2003), Comportamiento humano en el trabajo, México, Mc Graw Hill.

Heider, F. (1958) The Psychology of Interpersonal Relations. New York, USA: Wiley.

Kelley, H. H. (1972). Attribution in social interaction. In E. E. Jones, D. Kanouse, H. Kelley, R. Nisbett, S. 
Morgana (2004), Técnicas de la comunicación social. Percepción social, disponible en URL: www.ilustrados.com (recuperado el 25/08/2005)

Prieto N. L. (2003), Autoeficacia, recursos para la investigación, disponible en http://www.des.emory.edu/mfp/PrietoRecursos.html.

Trianes M. V. (2002), Niños con estrés, México, Alfa Omega-Narcea.

Weiner, B. (1974). “An attributional interpretation of expectancy-value theory”. En B. Weiner (Ed.), Cognitive views of human motivation. New York, USA: Academic Press.

Weiner, B. (1986). "Attribution, emotion, and action". En R.M. Sorrentino y E.T. Higgins (Eds.), Handbook of motivationa and cognition. Foundations of social behavior. New York, USA: John Wiley. 